\title{
Magnetic Resonance Imaging Findings in Small Patella Syndrome
}

\author{
Hyoung-Soo Kim, $\mathrm{MD}^{1}$, Jeong-Hyun Yoo, $\mathrm{MD}^{1}$, Noh-Hyuck Park, $\mathrm{MD}^{2}$, Jun-Hee Chang, $\mathrm{MD}^{1}$, \\ Yun-Seong Ban, $\mathrm{MD}^{1}$, and Sang-Heon Song, $\mathrm{MD}^{1}$ \\ Departments of ${ }^{1}$ Orthopaedic Surgery and ${ }^{2}$ Radiology, Myongji Hospital, Seonam University College of Medicine, Goyang, Korea
}

\begin{abstract}
Small patella syndrome (SPS) is characterized by aplasia or hypoplasia of the patella and pelvic girdle abnormalities, including bilateral absence or delayed ossification of the ischiopubic junction and infra-acetabular axe-cut notches. Here, we report a case of SPS in a 26-year-old female. Magnetic resonance image (MRI) showed a small patella with thick eccentric non-ossified patellar cartilage and femoral trochlear dysplasia with hypoplastic patellar undersurface. To our knowledge, this is the first report of MRI findings in SPS. MRI findings could be clinically relevant because elongation of the medial patellofemoral ligament and trochlear dysplasia with eccentric non-ossified patellar cartilage might lead to patellofemoral maltracking with an osteochondral lesion or acute dislocation or an extensor mechanism injury. Though the patient presented in this case report only had a gastrocnemius injury at the origin site, physicians should carefully examine abnormalities with MRI when an SPS patient has a trauma to the knee.
\end{abstract}

Keywords: Small patella syndrome, Magnetic resonance imaging

Small patella syndrome (SPS) is a rare autosomal dominant disorder due to mutations in the TBX4 gene ${ }^{1)}$, characterized by the absence or hypoplasia of the patella with various anomalies of the pelvis and feet including hypoplasia of the ischium, irregular ischial ossification at the ischiopubic synchondrosis, flat-foot or club-foot deformity, and brachydactyly. The syndrome was described first by Scott and Taor ${ }^{2)}$ in 1979: several cases of SPS accompanied by intermittent knee pain and recurrent patella dislocations were presented in the literature ${ }^{2)}$. However, there is no available literature addressing magnetic resonance imaging (MRI) findings of the knee in SPS patients. We report a patient with the SPS and demonstrate radiographic features and specific MRI

Received January 30, 2015; Revised April 28, 2015;

Accepted May 20, 2015

Correspondence to: Sang-Heon Song, MD

Department of Orthopaedic Surgery, Myongji Hospital, Seonam

University College of Medicine, 55 Hwasu-ro 14beon-gil, Deokyang-gu, Goyang 10475, Korea

Tel: +82-31-810-5424, Fax: +82-31-969-0500

E-mail: sh.gabriel.song@gmail.com

This is an Open Access article distributed under the terms of the Creative Commons Attribution Non-Commercial License (http://creativecommons.org/licenses/by-nc/4.0/) which permits unrestricted non-commercial use, distribution, and reproduction in any medium, provided the original work is properly cited. findings under the approval of the Institutional Review Board of this hospital with patient informed consent.

\section{Case Report}

A 26-year-old female patient presented with left knee pain after slip down while walking. On a physical examination, mild swelling and tenderness were identified in the medial aspect of the left knee. The power of quadriceps muscles was intact but she complained of pain during flexion-extension. The range of motion was limited due to pain: $90^{\circ}$ flexion and $-10^{\circ}$ extension. Grossly, patellofemoral maltracking was not identified but the patella size of both knees was smaller than normal.

Plain radiographs showed small patellae with abnormal morphology and hypoplastic medial femoral condyles in both knees (Fig. 1). So, we decided to undertake further assessment using MRI.

MRI images showed a partial rupture of the gastrocnemius medial head, but the meniscus and cruciate ligaments were intact (Fig. 2A). The sagittal fat-suppressed T2-weighted image showed thinning of the proximal patellar tendon and non-visualization of the articular cartilage in the patellofemoral compartment (Fig. 2A). The axial fat-suppressed T2-weighted image showed a small 

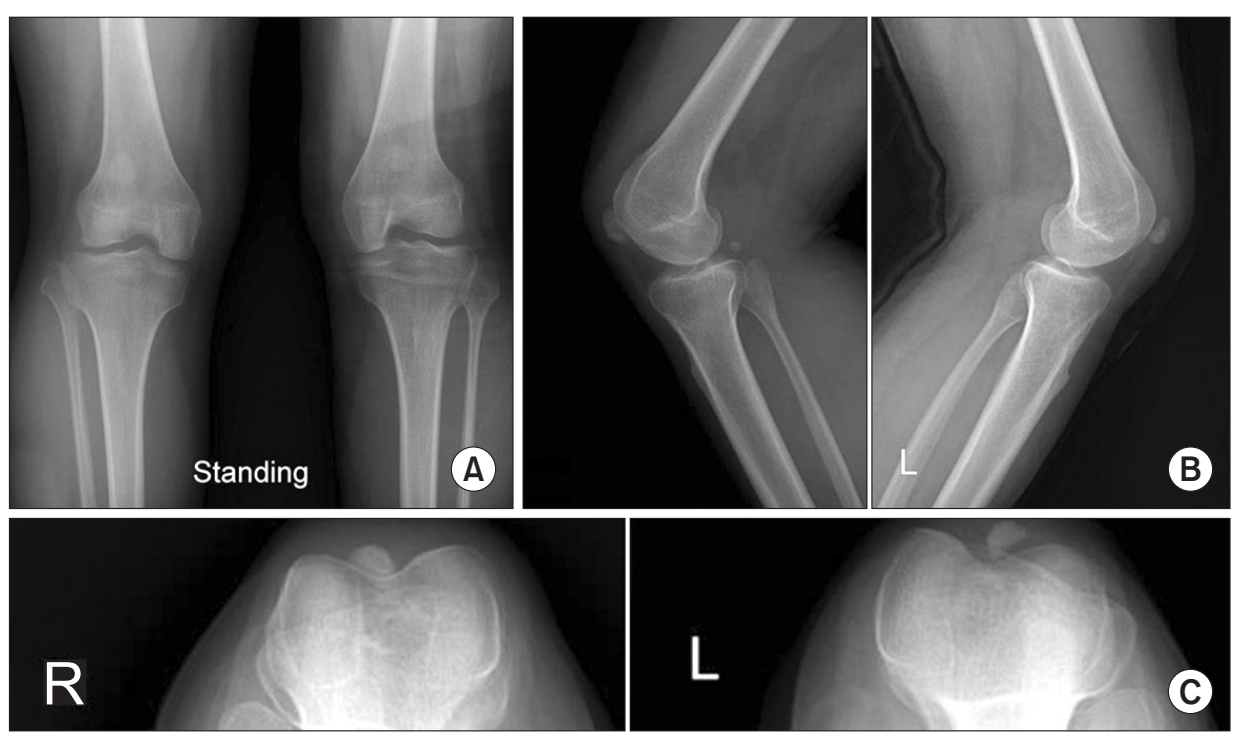

Fig. 1. (A) Standing anteroposterior radiograph of both knees showing small patellae with abnormal morphology. (B) Lateral radiographs of both knees showing small patellae. (C) Skyline Views of both knees showing small patellae. R: right, L: left.
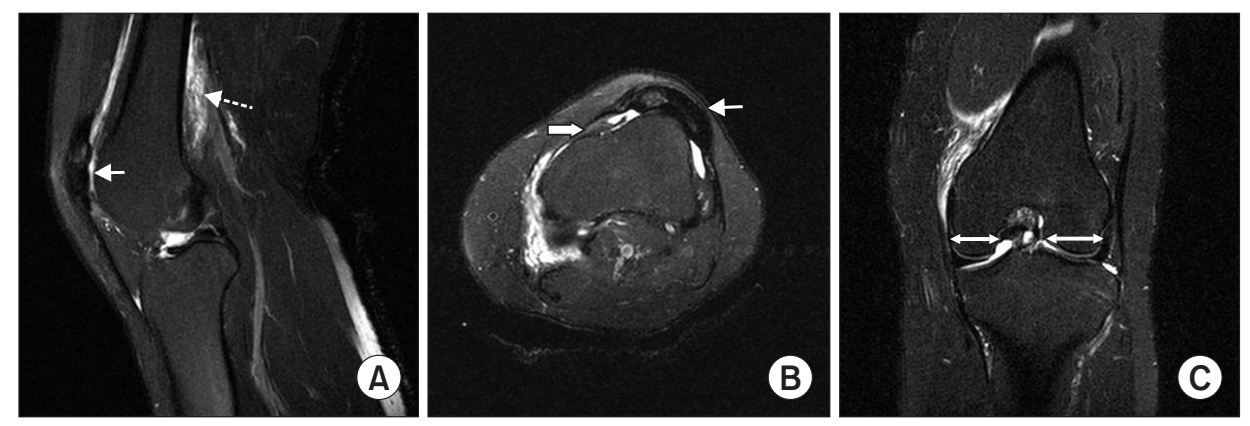

Fig. 2. (A) Sagittal fat-suppressed T2-weighted image revealing absence of articular cartilage of the patella undersurface (white arrow) and ruptured medial head of the gastrocnemius muscle (dotted arrow). (B) Axial fat-suppressed T2-weighted image revealing thick eccentric non-ossified patellar cartilage (arrows), elongated medial patellofemoral ligament (open arrows), and dysplastic trochlea. (C) Coronal fat-suppressed T2-weighted image revealing hypoplastic medial femoral condyle (arrow; width, $1.96 \mathrm{~cm}$ ) compared to the lateral condyle (arrow; width, $2.39 \mathrm{~cm}$ ).

patella $(1.0 \times 0.6 \times 1.5 \mathrm{~cm}$ in size) with lateral displacement, thick eccentric non-ossified patellar cartilage with central high signal portion, and elongated medial patellofemoral ligament with a tear at the femoral attachment site. Also, the trochlear groove was shallow (trochlear depth, $0.2 \mathrm{~cm}$ ), suggestive of trochlear dysplasia. Normal morphology of the medial and lateral facets on the posterior surface of the patella was not found (Fig. 2B). And the coronal fat-suppressed T2-weighted image showed a hypoplastic width of the medial femoral condyle (medial femoral condyle, $1.96 \mathrm{~cm}$; lateral femoral condyle, $2.39 \mathrm{~cm}$ ) (Fig. 2C).

Additional plain radiographs were taken for the evaluation of incidental findings of the small patella. The reported radiographic changes of SPS are hypoplastic or absent patella with pelvic and foot abnormalities ${ }^{3}$. Pelvic abnormalities include absent/delayed/ irregular ischial ossification at the ischiopubic synchondrosis, infra-acetabular axe-cut notches, high iliac angles, large femoral heads with narrowed femoral necks, coxa valga and vara, and hypoplastic lesser trochanters. Foot anomalies include widened first web space between toes, flat-foot or club-foot deformity, brachydactyly, and tarsal coalition. Some specific bony changes consistent with the diagnostic features of SPS were identified in the patient's knee, pelvis and foot. Hypoplastic ossification of the ischiopubic junction was observed on the pelvis radiograph, but an infra-acetabular axe-cut notch or a large femoral head with narrowed femoral neck was not observed (Fig. 3). Increases in the web space between the first and second toes (an intermetatarsal angle of $18^{\circ}$ for the right foot and an intermetatarsal angle of $19^{\circ}$ for the left foot) were observed along with the short fourth and fifth rays on the foot radiographs (Fig. 4). However, pes planus was not observed.

We recommended additional genogram survey to the patient because findings suggestive of SPS were observed. However, fur- 


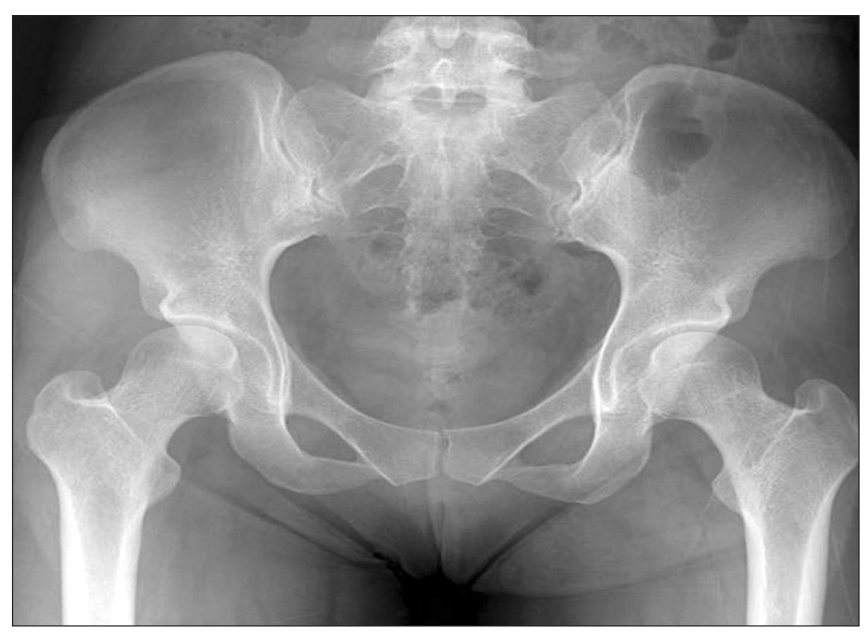

Fig. 3. Anteroposterior radiograph of the pelvis showing hypoplastic ossification of the ischiopubic junction.

ther examinations could not be done due to the patient's refusal. The patient's height and weight were about $152 \mathrm{~cm}$ and $47 \mathrm{~kg}$, respectively, showing less than 5th percentile values of stature. There were no other morphological changes such as facial dysmorphism or nail dysplasia on her appearance. She said that her patellae had been found to be smaller than normal people in the past but did not present with related complaints or additional clinical symptoms. The patient's family history revealed that she is the first of two daughters, and no one had been diagnosed with SPS.

The patient was treated conservatively with non-steroidal antiinflammatory drugs and temporary immobilization due to partial injury to the gastrocnemius muscle. After 3 weeks of conservative treatment, her symptoms improved and she returned to her normal life with pain-free full range of motion.

\section{Discussion}

SPS, also known as ischiopatella dysplasia, coxo-podo-patellar syndrome, ischio-pubic-patella syndrome or Scott-Taor syndrome, is a rare autosomal dominant disorder characterized by the absence or hypoplasia of the patella and various anomalies of the pelvis and feet.

The syndrome was described first by Scott and Taor ${ }^{2}$ in 1979. In their study, among twelve members of a family affected by patellar hypoplasia/aplasia, seven individuals showed abnormalities of the pelvic girdle and upper femora. In 1981, Vanek ${ }^{4)}$ reported 3 familial cases that showed infra-acetabular axe-cut notches besides hypoplastic patellae. Vanek ${ }^{4)}$ proposed the name of ischiopatellar dysplasia for the syndrome. The pedigree was consistent
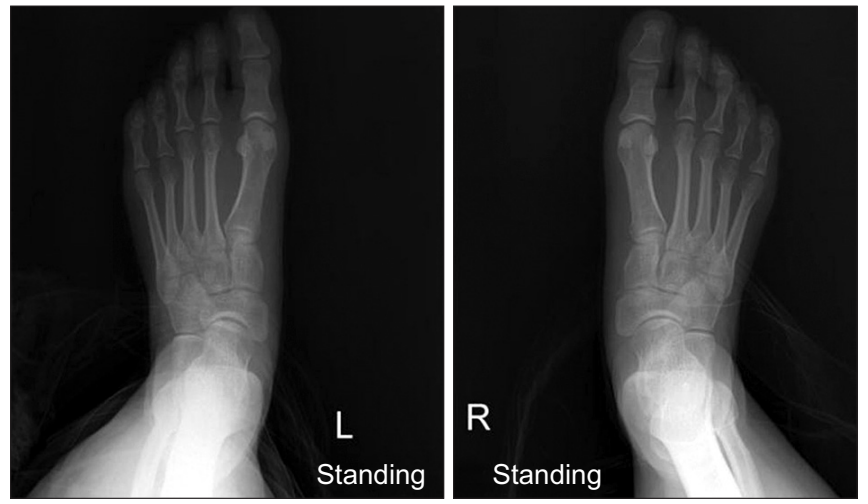

Fig. 4. Standing anteroposterior radiographs of both feet showing increased web space between the 1st and 2nd toes: intermetatarsal angle of $18^{\circ}$ for the right (R) foot and intermetatarsal angle of $19^{\circ}$ for the left (L) foot.

with autosomal dominant inheritance. In 1985, fifteen patients with a similar disorder were described by Morin et al. ${ }^{5)}$ under the diagnosis of coxo-podo-patellaire syndrome. Of those, eleven patients whose feet had been examined showed an increased space between the first and second toes. In most of them, short 4th and 5th toes and flat feet were also noted. In 1995, Kozlowski and Nelson ${ }^{3)}$ reported two sporadic cases that showed more generalized bone changes and dysmorphic features. The two patients had a number of facial dysmorphic features with prominent forehead, posteriorly angulated ears, broad nasal bridge, long philtrum, high plate, and prominent lower lip.

The diagnostic radiographic changes of SPS are hypoplastic or absent patella with minor hypoplastic/dysplastic knee changes and pelvic abnormalities ${ }^{3}$. Pelvic abnormalities include absent/ delayed/irregular ischial ossification at the ischiopubic synchondrosis, infra-acetabular axe-cut notches, high iliac angles, large femoral heads with narrowed femoral necks, coxa valga and vara, and hypoplastic lesser trochanters. Other major features include anomalies of the feet, a wide space between the 1st and 2nd toes, flat-foot or club-foot deformity, brachydactyly, other hypoplastic/ dysplastic changes, and tarsal coalition. Our patient showed bony changes with diagnostic features of SPS in the knee, pelvis, and foot.

According to our literature review, this is the first report of MRI findings in SPS. In this study, MRI scans showed thick eccentric non-ossified patellar cartilage, elongated medial patellofemoral ligament, hypoplastic medial femoral condyle, and trochlear dysplasia. Normal morphology of medial and lateral facets on the posterior surface of the patella was not found. Also, thinning of the proximal patellar tendon and non-visualization of the articular cartilage of the patellar undersurface were identified. 
These findings could be clinically relevant because elongation of the medial patellofemoral ligament and trochlear dysplasia with eccentric non-ossified patellar cartilage might lead to patellofemoral maltracking with an osteochondral lesion or an acute dislocation or an extensor mechanism injury. Though the patient in this study only had a gastrocnemius injury at the origin site, physicians should carefully examine abnormalities with MRI when an SPS patient has a trauma to the knee.

The skeletal dysplasia resulted from the mutations in the human homologue of the TBX4 gene ${ }^{1)}$. The SPS phenotype suggests that human TBX4 mutations do not affect early stages of limb development, such as limb-bud initiation, but do have a profound impact on the later stages. The late influence of TBX4 mutations is manifested by patellar aplasia/hypoplasia and anomalies of the posterior structures of the foot, such as the space between the first and second toes and short fourth and fifth rays. Completion of ischiopubic formation is another very late process in human development, which is disrupted in individuals with SPS.

With respect to the need of evaluation of systemic organ dysfunction in kidney or neuropathy, SPS should be differentiated from absent or hypoplastic patellae. Nail patella syndrome (NPS) is an autosomal dominant disorder due to mutations in the LMX1B gene ${ }^{6}$. Nail dysplasia is seen in over $90 \%$ of patients. Other features include hypoplasia or absent patellae, a single iliac horn on each side extending backwards and outwards from the mid part of the iliac bone, and elbow deformities, mainly hypoplasia of the radial head and/or capitellum causing subluxation or dislocation ${ }^{7}$. One of the most serious manifestations of NPS is kidney disease since approximately one third of patients develop renal involvement including glomerulonephritis or nephrotic syndrome and their complications ${ }^{8}$. Our patient did not have nail dysplasia, kidney disease, or iliac horns. Moreover, a small or absent patella may be seen as an isolated finding. It may be either unilateral or bilateral and may be familial, being transmitted in an autosomal-dominant mode patella aplasia-hypoplasia (PTLAH) ${ }^{9}$. In PTLAH, hereditary motor and sensory neuropathy could be presented and congenital aplasia/hypoplasia of the patella are also clinical key features. Finally, very rare causes of absent or hypoplastic patellae include arthrogryposis-like Kuskokwim syndrome, trisomy 8, and Coffin-Siris syndrome ${ }^{10)}$.

In conclusion, SPS is an autosomal-dominant bony dysplasia of the pelvis, knees, and feet. The remaining skeleton shows minor changes that can be easily overlooked. Therefore, it is important to perform a complete skeletal survey on all patients with absent or hypoplastic patellae. Also, it is advised to perform MRI evaluation to find out any acute patellofemoral injuries when an SPS patient has a trauma to the knee.

\section{Conflict of Interest}

No potential conflict of interest relevant to this article was reported.

\section{References}

1. Bongers EM, Duijf PH, van Beersum SE, Schoots J, Van Kampen A, Burckhardt A, Hamel BC, Losan F, Hoefsloot LH, Yntema HG, Knoers NV, van Bokhoven H. Mutations in the human TBX4 gene cause small patella syndrome. Am J Hum Genet. 2004;74:1239-48.

2. Scott JE, Taor WS. The "small patella" syndrome. J Bone Joint Surg Br. 1979;61:172-5.

3. Kozlowski K, Nelson J. Small patella syndrome. Am J Med Genet. 1995;57:558-61.

4. Vanek J. Ischiopatellar dysplasia (Scott and Taor's syndrome of the small patella). Rofo. 1981;135:354-6.

5. Morin P, Vielpeau C, Fournier L, Denizet D. The coxopodopatellar syndrome. J Radiol. 1985;66:441-6.

6. Dreyer SD, Zhou G, Baldini A, Winterpacht A, Zabel B, Cole W, Johnson RL, Lee B. Mutations in LMX1B cause abnormal skeletal patterning and renal dysplasia in nail patella syndrome. Nat Genet. 1998;19:47-50.

7. Sweeney E, Fryer A, Mountford R, Green A, McIntosh I. Nail patella syndrome: a review of the phenotype aided by developmental biology. J Med Genet. 2003;40:153-62.

8. Lemley KV. Kidney disease in nail-patella syndrome. Pediatr Nephrol. 2009;24:2345-54.

9. Bernhang AM, Levine SA. Familial absence of the patella. J Bone Joint Surg Am. 1973;55:1088-90.

10. Azouz EM, Kozlowski K. Small patella syndrome: a bone dysplasia to recognize and differentiate from the nail-patella syndrome. Pediatr Radiol. 1997;27:432-5. 